\title{
Review of external ocular compression: clinical applications of the ocular pressure estimator
}

\section{Michael S Korenfeld ${ }^{1,2}$ \\ David K Dueker ${ }^{3}$}

'Comprehensive Eye Care, Ltd. Washington, MO, USA; ${ }^{2}$ Washington University Department of

Ophthalmology and Visual Sciences, St Louis, MO, USA; ${ }^{3}$ Ophthalmology, Hamad Medical Corporation, Doha, Qatar

Video abstract

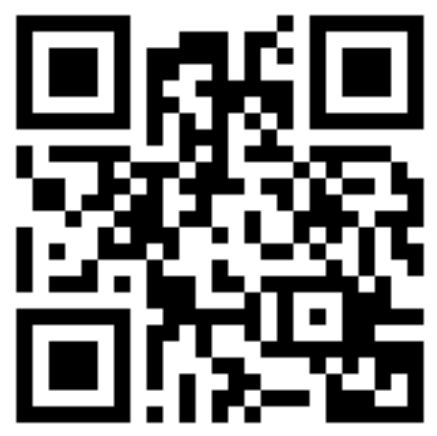

Point your SmartPhone at the code above. If you have a QR code reader the video abstract will appear. Or use: http://youtu.be/dpPOykLi23Y
Correspondence: Michael S Korenfeld Comprehensive Eye Care, Ltd. and Washington University Department of Ophthalmology and Visual Sciences, 90I East 3rd Street, Washington, MO 63090, USA

Tel +l 6363903999

Fax +l 6363903959

Email michaelkorenfeld@hotmail.com
This article was published in the following Dove Press journal:

Clinical Ophthalmology

23 February 2016

Number of times this article has been viewed

Purpose: The authors have previously validated an Ocular Pressure Estimator (OPE) that can estimate the intraocular pressure (IOP) during external ocular compression (EOC). The authors now apply the OPE in clinical states where EOC is clinically important. The original work is described for two periods of risk: during sleep and during the digital ocular massage (DOM) maneuver used by surgeons after trabeculectomy to keep the operation functional. Other periods of risk for external ocular compression are then reviewed.

Methods: The first protocol estimated the IOP in the dependent eye during simulated sleep. Subjects had their IOPs initially measured in an upright-seated position, immediately upon assuming a right eye dependent side sleeping position (with nothing contacting the eye), and then 5 minutes later while still in this position. While maintaining this position, the fluid filled bladder of the OPE was then placed between the subject's closed eye and a pillow during simulated sleep. The IOP was continuously estimated in this position for 5 minutes. The subjects then had the IOP measured in both eyes in an upright-seated position. The second protocol determined if a larger vertical cup-to-disc ratio was more common on the side that patients reported they preferred to sleep on. The hypothesis was that chronic asymmetric, compression induced, elevations of IOP during sleep would be associated with otherwise unexplained asymmetry of the vertical cup-to-disc ratio. The third protocol assessed the IOP during DOM. The OPE was used to characterize the IOP produced during the DOM maneuver of five glaucoma surgeons. After this, $90 \mathrm{mmHg}$ was chosen as a target pressure for DOM. The surgeons were then verbally coached during three additional compressions. After a 5-minute period, the surgeons were asked to reproduce this targeted IOP during subsequent compressions.

Results: The mean IOP during the "sleep session" was $22 \pm 5 \mathrm{mmHg}$ (SEM). The mean peak pressure was $40 \pm 11 \mathrm{mmHg}$ (SEM) and the mean trough pressure was $15 \pm 2 \mathrm{mmHg}$ (SEM). There was a $78 \%$ agreement between the eye that was reported to be dependent during sleep and the eye with the larger vertical cup-to-disc ratio, for eyes with at least a 0.10 cup-to-disc ratio difference, $P=0.001, \mathrm{n}=137$. The OPE estimated an average induced IOP during typical DOM of $104 \pm 8 \mathrm{mmHg}$ (SEM), with each compression having an average range of $17 \pm 3 \mathrm{mmHg}$ (SEM). After coaching, and a 5-minute waiting period, the average induced IOP reduced to $95 \pm 3 \mathrm{mmHg}$ (SEM) with a reduced average range of IOP to $11 \pm 1 \mathrm{mmHg}$.

Conclusion: The OPE was successfully used to estimate the IOP while subjects experienced EOC during normal sleep postures. These EOC-induced elevations of IOP were considerable, and likely contribute to significant ocular pathology, not only for glaucoma, but for retinal vascular occlusive diseases, retinal vascular leakage, and the induction of the ocular-cardiac reflex in infants, as well. The correlation of a larger vertical cup-to-disc ratio in patients with a sleep posture preference suggests a causal relationship, since patients with other conditions known to be associated with cup-to disc ratio asymmetry were excluded from this study. The OPE is a useful device to teach DOM to surgeons and patients for home use.

Keywords: sleep posture, intraocular pressure, nocturnal, cup-to-disc ratio asymmetry, glaucoma, digital ocular massage 


\section{Introduction}

Elevated intraocular pressure (IOP) is an unambiguous and established risk factor for glaucomatous optic nerve damage. Historically, the IOP in live humans has been estimated with many different devices, all of which directly or indirectly apply force to the globe while the eyelids are open, and the devices have access to the ocular surface. There are instances when the IOP is clinically relevant, but existing devices that estimate IOP are impossible to use. These clinical settings occur when patients' eyes are closed, eg, during sleep, while rubbing the eyes, and during the digital ocular massage (DOM) maneuver that is used after trabeculectomy surgery to maintain a functional bleb. In this paper, the following original protocols were performed to characterize the IOP transients induced during periods of external ocular compression.

The authors have invented, constructed, and validated such a device. The details of the device's construction and its validation on a manometrically cannulated human cadaver eye and an in vivo pig eye are thoroughly described. ${ }^{1}$ Briefly, the ocular pressure estimator (OPE) was designed around the principle that if two vessels with similar wall compliances are placed in contact with one another within a confined space, and an external force is applied to one of the vessels, the pressure inside both vessels becomes the same. For example, if two tennis balls are placed into a can, and an external force pushes on the outermost ball, the pressure inside both balls becomes the same. The OPE is a fluid-filled device with a wall compliance that is similar to the eye wall: flexible, but relatively inelastic. The OPE has a thin fluid-filled bladder that is approximately $1.0 \times 1.5 \mathrm{~cm}$ in size, and $2-3 \mathrm{~mm}$ thick when filled with water, and ready to use. A fluid continuum from the lumen of the bladder to the pressure transducer was established with high-pressure tubing that terminated in a three-way stopcock that was attached to the pressure transducer. The stopcock enabled the system to be filled with water and to be calibrated with a fluid column lifted above the transducer at different heights. The output of the transducer was amplified and sent to a strip chart recorder. Pressure transients experienced within the fluid-filled bladder (the portion of the OPE that is placed against the eye) were hydraulically transmitted to and sensed by the pressure transducer, remotely located from the fluid-filled bladder. Once the OPE was constructed, an analogous system was configured, only for the control setup, the device terminated in a needle for globe cannulation, instead of the fluid-filled bladder. The OPE was used for two of the clinical experiments described in this paper.

\section{Protocol I: external ocular compression during sleep (short-term exposure)}

1) Determination if simply changing positions from sitting to reclining on one side, without any contact with the dependent eye induces changes in IOP $(n=5)$.

2) Determination of induced IOP when the dependent eye is externally compressed when it comes into contact with a pillow $(\mathrm{n}=9)$.

\section{Protocol 2: external ocular compression during sleep (long-term exposure)}

1) Determining the correlation of vertical cup-to-disc ratio asymmetry, which has no obvious cause, to patients' reported preferred sleep posture that places their dependent eye on the same side that has the larger optic nerve cup $(n=137)$.

\section{Protocol 3: external ocular compression during DOM}

1) Determining the induced IOP elevations during the DOM maneuver using the OPE, and then using the OPE to teach surgeons to reproduce a reasonable experimental target IOP during DOM $(\mathrm{n}=5)$.

Glaucoma is currently thought to result from a derangement in the normal interrelationships of IOP, vascular perfusion of the optic nerve, and the mechanical integrity of the optic nerve and its surrounding support structures. For practical reasons, clinical evaluations of these variables and visual function have largely been limited to daytime. Many investigators have recognized the potential importance of fluctuations in ocular physiology at night, and descriptions of diurnal variations of aqueous flow, ${ }^{2-4} \mathrm{IOP}, 5,6$ and aqueous humor protein concentration ${ }^{7}$ have been published. The assessments of IOP during "sleep" "5,6 in all prior studies have focused on measurements by standard methods on subjects who were awakened for measurements. Distinctions have been made between the differential effects of sudden awakening versus gentle arousal on IOP during "sleep". 5,6 Severe unilateral visual loss has also been described in patients with normal blood pressures during general anesthesia; the visual loss was produced by ocular compression by the passive weight of the head while patients were positioned in the prone position. ${ }^{8}$ In a case report, an obese patient with unilateral floppy eyelid syndrome, keratoconus, and advanced glaucoma was described; ${ }^{9}$ this patient was reported to routinely sleep with "significant digital compression of the involved eye". 
The focus of the first protocol of this study was to ascertain whether the weight of the head, in association with certain sleeping postures, could transmit pressure to the orbit and raise the IOP to levels that would be considered clinically important in an awake state. The OPE was used to make these measurements.

The vertical cup-to-disc ratio is a common measurement that is used to characterize the presence, severity, and stability of glaucoma. As glaucoma progresses, the vertical cup-todisc ratio becomes larger. An enlarged cup-to-disc ratio is a characteristic feature of glaucomatous optic atrophy that is uncommon with other forms of optic atrophy. In the second protocol of this paper, the authors sought to determine whether patients who were first observed to have a measurable vertical cup-to-disc ratio asymmetry subsequently reported that they had a preferred sleep posture that put the side with the larger cup in a dependent position during their lifetimes. This protocol was designed to test the hypothesis that chronically elevated nocturnal elevations of IOP due to external compression in the dependent eye could produce asymmetric optic nerve damage, when all other potential causes for this had been excluded.

The potential for failure at different times after trabeculectomy has resulted in several strategies to maintain the patency of the bleb. One strategy is to compress or massage the globe through a closed lid, elevating the IOP, and mechanically forcing aqueous humor through the surgical wound. ${ }^{10-18}$ When successful, the forced passage of aqueous humor breaks weak adhesions in the outflow pathway and extends the bleb area. The usual endpoint of DOM is either visual elevation of the bleb with attendant reduction of the IOP, or simply a substantial diminution of the IOP. The technique usually involves cycles of externally applied pressure, followed by intervals of no pressure application. This technique allows the clinician to visualize the outcome of the effort, provides a moment of recovery for the patients who occasionally experience discomfort, and provides time for reperfusion of the eye. DOM is administered by surgeons during postoperative examinations and is often taught to patients for self-administration at home. There are currently no precise guidelines regarding the amount or duration of force required, except that it should be sufficient to lower the IOP and elevate, or extend, the filtration bleb. These are both endpoints that cannot be reliably assessed outside the physician's office. Additionally, the IOP induced during successful DOM is unknown.

The focus of the third protocol of this review paper was to use the OPE to quantify and characterize the IOP transients produced during DOM, as typically performed by several surgeons who utilize it in postoperative management. Once a reasonable experimental target pressure was established, the OPE was then used to teach these surgeons how to produce this targeted pressure during subsequent DOM maneuvers.

\section{Materials and methods}

Two approaches were taken to assess the potential impact of external ocular compression during sleep. The first protocol was designed to estimate IOP in the dependent eye during simulated "sleep". One hour before a simulated "sleep" session, five normotensive human volunteers had their IOP measured by Goldmann applanation tonometry. This was done 1 hour before simulated "sleep" to ensure that the anesthetic effect from measuring the IOP had dissipated, so the subjects could feel any discomfort that might be produced during ocular compressions during the "sleep session". Then, just before lying down, these subjects had their IOPs estimated by a Perkins tonometer in an erect sitting position in both eyes. They were then instructed to lie on a bed on their right side and not to have their dependent eye come in contact with any surface. The subjects' IOPs were measured with a Perkins tonometer immediately after assuming this side-sleeping posture and again 5 minutes after maintaining. This protocol was similar to the one described for the next experiment, except there was no external compressive pressure applied to either eye in this initial protocol.

Healthy ocular normotensive subjects were subsequently instructed to lie on a bed on their right side with their head on a pillow. Before the side of their face made contact with the pillow, the thin pressure sensing fluid-filled bladder portion of the OPE was placed over the closed eyelid and allowed to come to rest between the closed eyelid and the pillow. Care was taken to place the device on the eyelid over the globe, without contact with the bony orbit. The plastic surface of the device loosely stuck to the eyelid skin without the need for adhesives. Patients were instructed to assume a position that was a realistic sleep posture for them and to allow the fully relaxed weight of their head come to rest on the pillow, without forcing their head into it. Similar to the noncompressive protocol described earlier, the IOP was estimated by Goldmann applanation tonometry 1 hour before and within 1 minute after a 5-minute "sleep" session. Exophthalmometry readings were also recorded for all subjects.

The second protocol of this paper was designed to study the relationship of vertical cup-to-disc ratio asymmetry to a 
patient's reported sleep posture preference. It is postulated that years of external ocular compression during sleep, with clinically relevant increases of IOP, could result in a vertical cup-to-disc ratio asymmetry, if the patient had a preferred sleep posture preference with one eye being more consstently in a dependent position. The population for this arm of the study consisted of all patients contacted by one of the authors (MSK) during an 8-month period who fulfilled the following criteria.

Patients were initially identified by the presence of a difference in the cup-to-disc ratio between the two eyes. After a qualitative difference was established, quantification of the vertical cup-to-disc ratios was performed. Estimates were made using a $90 \mathrm{D}$ lens with a slit lamp through dilated pupils. Patients had to have an unambiguous disparity in the vertical cup-to-disc ratio asymmetry of greater than or equal to 0.1 to be included in this study. After the examiner documented the presence and degree of the cup-to-disc ratio asymmetry, a uniform series of questions were then asked as follows: "When you sleep, do you tend to sleep on our back, your tummy, or your side?" If patients reported that they were strictly back sleepers they were not eligible for this study; the assumption being that if they were correct about their sleep posture, there would be no opportunity for the weight of the head to be transmitted to the globe. For the purposes of this study, sleeping preferentially on one's left side was considered equal in potential risk to sleeping in a prone position with one's head turned to the right; the left eye is dependent in both instances. When possible, a patient's spouse was asked to corroborate the patient's stated sleep preference. In general, there was excellent spouse-patient agreement, ${ }^{19}$ and if the responses were opposite, the patient was excluded from this study. After initial questioning, patients would occasionally state that they always slept on a particular side. This was usually secondary to a cardiorespiratory or orthopedic condition..$^{20,21}$ More commonly, patients designated themselves as side sleepers, prone sleepers, or both. To ascertain side preference, the following question was asked: "When you sleep on your side, do you have a favorite side, or do you flip from side to side? You do not have to have a favorite side". The latter questions were designed to identify those patients with true side preferences while providing them the verbal cue that not having a preference was acceptable. If patients preferred a prone position, they were asked, "When you sleep on your tummy, do you turn your head more often to the left or the right, or do you really have no preference for one side over the other? You do not have to have a favorite side". For all patients identified as having cup-to-disc ratio asymmetry and a sleep position preference, a record was made of age, sex, sleep position preference, the vertical cup-to-disc ratio of both eyes, and the IOPs (Goldmann applanation tonometry). All patients with a greater than $2 \mathrm{mmHg}$ difference of IOP between the two eyes were excluded; patients being treated for bilateral glaucoma had to satisfy this criterion before and consistently during treatment. Patients with asymmetric ocular disease that would differentially affect the IOP (ie, unilateral pseudoexfoliation, trauma, pigment dispersion) or the number of optic nerve head axons (ie, retinal and optic nerve vascular occlusions, retinal laser treatment) were excluded. Patients with ambiguous vertical cup-to-disc ratios were also excluded; these included gradually sloping rims, irregularly shaped cups and optic disc abnormalities. Patients were also excluded if they had presurgical ammetropia of $\geq 2 \mathrm{D}$.

In the third protocol of this study, the OPE was used to estimate the IOP transients produced during DOM. Five glaucoma surgeons, experienced in the postoperative use of DOM, participated in these experiments. Unoperated, ocular normotensive human beings were used as subjects. Initially, each surgeon was asked to apply the fluid-filled bladder to the subject's closed eyelid and apply digital pressure through it to the patient's globe, in a manner that reproduced their typical DOM technique. They were encouraged to produce four compressions, each for a 10 -second period. They were instructed to maintain a constant induced pressure during each compression. These initial compressions were done while the surgeons were masked to the pressures they were producing. After the five surgeons had produced their ideal personal compression pressures, it was determined that all surgeons had produced a value of at least $90 \mathrm{mmHg}$. As a result of this, $90 \mathrm{mmHg}$ was designated as a reasonable experimental "target" pressure to achieve during the next experiment. Each surgeon was again asked to perform DOM; only this time, one investigator provided verbal cues to "teach" the surgeon to feel the targeted IOP during three additional ocular compressions, while the "teacher" visualized the induced pressures on the strip chart recorder, and the surgeon was visually masked to them. After 5 minutes, the surgeons were again asked to perform DOM in a masked manner, and try to repeat the target pressure of $90 \mathrm{mmHg}$ that they had been taught to produce by coaching. Ethical and legal approval was obtained prior to the commencement of the study. This was provided by the University of MissouriColumbia, Columbia, Missouri. All experiments performed in support of this paper were performed in accordance with the tenets of the Declaration of Helsinki and followed relevant institutional and national guidelines and regulations. 
Table I Results of the estimates of IOP in normal human volunteers while erect, just after assuming a reclining posture on the right side, and after 5 minutes in this reclining posture

\begin{tabular}{llll}
\hline Patient & Erect & On side & On side, after 5 minutes \\
\hline I) $25, \mathrm{~F}$ & $16 / 15$ & $16 / 16$ & $16 / 16$ \\
2) $32, \mathrm{~F}$ & $10 / 11 \mathrm{I}$ & $9 / 1 \mathrm{I}$ & $10 / 11$ \\
3) $29, \mathrm{M}$ & $13 / 14$ & $14 / 14$ & $14 / 13$ \\
4) $25, \mathrm{M}$ & $1 \mathrm{I} / 1 \mathrm{I}$ & $12 / 12$ & $12 / 12$ \\
5) $27, \mathrm{~F}$ & $15 / 15$ & $16 / 16$ & $16 / 16$ \\
\hline
\end{tabular}

Notes: No contact was made with the dependent right eye except for during the IOP measurements that were all made with the same Perkins tonometer. IOP measurement is in $\mathrm{mmHg}$. Patient age in years and sex are noted, $n=5$.

Abbreviations: IOP, intraocular pressure; $F$, female; $M$, male.

All patients provided written informed consent after receiving a thorough explanation of the protocol.

\section{Results}

\section{Protocol I, Experiment I}

The IOP was estimated in an erect and a right-sided reclining posture in five healthy young adults to determine whether assuming the latter posture without eye contact contributed to the IOP elevations observed during right-sided posture with eye contact (Table 1). There was no significant increase in IOP attributed to simply lying on the right side without dependent eye contact.

\section{Protocol I, Experiment 2}

In general, the IOP graphs were similar to tonographic tracings, showing fine respiratory associated pressure oscillations superimposed on a gradually decreasing pressure. A representative tracing is seen in Figure 1. The mean IOP during the 5-minute test period for all subjects was $22 \pm 5 \mathrm{mmHg}$ (SEM), $n=9$ (Table 2). The highest and lowest IOPs for different subjects varied considerably. The mean peak IOP during the test period was $40 \pm 11 \mathrm{mmHg}$ (SEM) and the mean trough IOP was $15 \pm 2 \mathrm{mmHg}$ (SEM). One subject had an estimated pressure that averaged $57 \mathrm{mmHg}$ and which approached $100 \mathrm{mmHg}$ (after a position switch) for the last minute and a half of testing; this subject was almost asleep when the testing period was stopped.

In all subjects but one (subject F), there was a drop in the IOP after the sleep session in the dependent eye only (Table 2). This drop in IOP was judged to be a further confirmation that eye contact with the pillow had caused an orbital compression. In the one case (subject F) where pre- and post-"sleep" IOPs were the same, the estimated IOP during side-sleeping was less than the Goldmann IOP during the entire simulated "sleep" session. These data for subject $F$ were interpreted to be the result of an incomplete mechanical coupling of the closed eye and the bedding pillow, despite the observation of proper positioning of the fluid-filled bladder on the eyelid before and just after the "sleep" session. There was no apparent relationship of the exophthalmometry readings to changes of IOP observed during side sleeping.

\section{Protocol 2, Experiment I}

A total of 137 patients were studied in the sleep position preference association with cup-to-disc ratio asymmetry protocol. There were 97 males and 40 females. The mean age was $62 \pm 16$ years (SD) for men and $56 \pm 18$ years (SD) for women. The age range was from 14 to 90 years. The patients studied were from the Harry S. Truman Memorial Veteran's Hospital and the University of Missouri Eye Clinic. The analysis of the cup-to-disc ratios in this study assigned equal weight to the difference between a 0.1 and a 0.2 cup and to that between 0.7 and a 0.8 cup, both with a difference of 0.1 . The difference between the latter pair of cups,

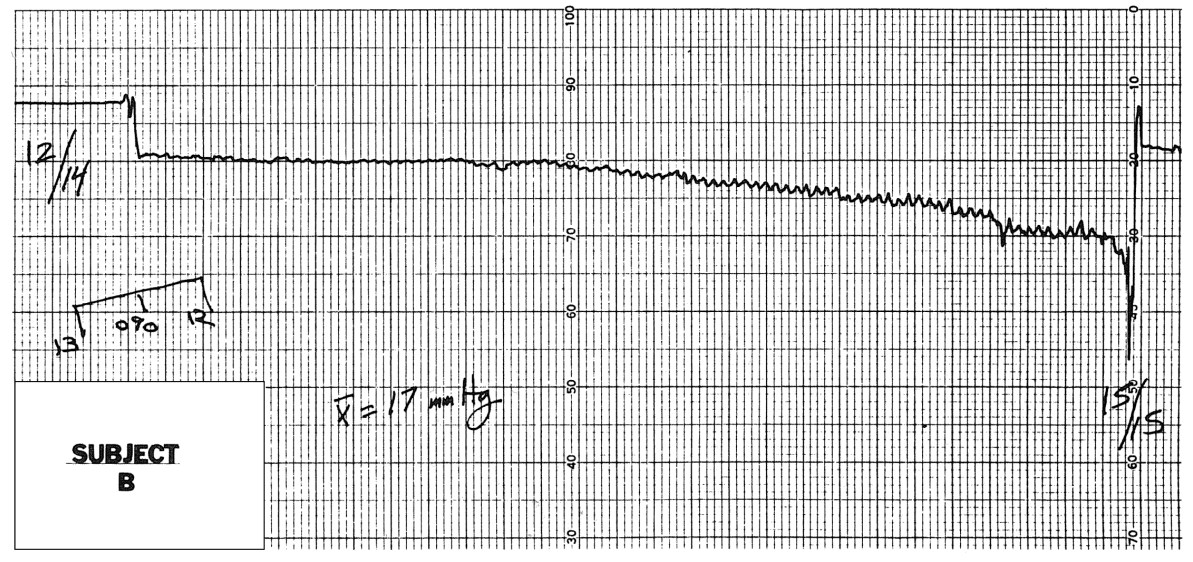

Figure I The tracing of subject B during the 5-minute simulated "sleep session".

Notes: The initial rise and slow drift toward a lower IOP plateau was the most characteristic pattern observed among the other subjects. Note the fine superimposed respiratory pressure variations.

Abbreviation: IOP, intraocular pressure. 
Table 2 Results from of the all subjects during the 5-minute simulated "sleep session", $\mathrm{n}=9$

\begin{tabular}{|c|c|c|c|c|c|}
\hline \multirow[t]{2}{*}{ Subject } & \multicolumn{4}{|c|}{ Intraocular pressures } & \multirow{2}{*}{$\begin{array}{l}\text { Hertel measurements } \\
\text { right-left eye }\end{array}$} \\
\hline & Presleep & Postsleep & Mean during sleep & Peak/trough during sleep & \\
\hline A & $9 / 10$ & $6 / 11$ & 57 & $100 / 19$ & $14-14$ \\
\hline B & $15 / 15$ & $12 / 14$ & 17 & $30 / 13$ & $13-12$ \\
\hline C & $16 / 16$ & $9 / 16$ & 19 & $95 / 14$ & $17-16$ \\
\hline D & $18 / 20$ & $16 / 21$ & 27 & $32 / 24$ & $19-20$ \\
\hline$E$ & $8 / 10$ & $6 / 10$ & 21 & $25 / 15$ & $18-18$ \\
\hline $\mathrm{F}$ & $14 / 14$ & $14 / 14$ & 6 & $12 / 05$ & $17-17$ \\
\hline G & $10 / 10$ & $6 / 10$ & 15 & $21 / 13$ & $13-12$ \\
\hline $\mathrm{H}$ & $14 / 14$ & $10 / 14$ & 14 & $15 / 13$ & $16-15$ \\
\hline I & $18 / 16$ & $12 / 18$ & 21 & $30 / 16$ & $19-19$ \\
\hline
\end{tabular}

Notes: Right eye/left eye, right eye is the dependent eye. Pre- and post-"sleep" IOPs were measured using the same Goldmann applanation tonometer. All other IOPs were estimated with the fluid-filled bladder and the OPE. The mean "sleep" IOP is a time weighted average during the 5-minute simulated "sleep session". All IOP measurements are in $\mathrm{mmHg}$ and Hertel exophthalmometry measurements are in millimeters.

Abbreviations: IOP, intraocular pressure; OPE, ocular pressure estimator.

however, may represent a more pathologic situation since the number of axons lost as a cup progresses from 0.7 to 0.8 is probably greater than when a cup progresses from 0.1 to 0.2 , since there are likely more optic nerve axons in an annulus of larger diameter.

When the variable of a sleep side preference was considered along with age and sex in a multiple regression analysis (Statistical Analysis System software, version 6; SAS Institute Inc., Cary, NC, USA), age and sex did not contribute significantly to the linear regression model, although there was a trend for the magnitude of the cup-to-disc ratio asymmetry to increase with increasing age. The data relating sleep side preference and cup-to-disc ratio asymmetry were evaluated in subgroups. The subgroups were created by pooling patients who were estimated to have at least a $0.10,0.15,0.20$, and 0.25 difference in the cup-to-disc ratio between the two eyes. Therefore, all 137 study subjects had a cup-to-disc ratio asymmetry of $\geq 0.1$. A smaller subset of the total patients had a cup-to-disc ratio that was $\geq 0.15$, and all subjects in this group were also included in the group that had a cup-to-disc ratio asymmetry of $\geq 0.1$. This strategy was applied to other degrees of cup-to-disc ratio asymmetry, so that all subjects in the group with a cup-to-disc ratio asymmetry of $\geq 0.25$ were included in all the groups with the smaller cup-to-disc ratio asymmetry degrees. There was a $78 \%-85 \%$ agreement between the side with the larger cup-to-disc ratio and the side with eye dependency during sleep. This degree of agreement was statistically significant for all age groups (McNemar test, ${ }^{22} P \leq 0.01$ for all four subgroups; Table 3 and Figure 2). The findings in this arm of the study were similar for men and women. In this study population, $63 \%$ preferred sleeping on their right sides, a statistically significant difference, $P=0.0013$, by one sample test of proportions.

Table 3 A statistically significant relationship was found between the preferred sleep posture and the side with the larger cup-to-disc ratio using a McNemar test for paired data with dichotomous outcomes

\begin{tabular}{|c|c|c|c|c|c|c|}
\hline & \multirow[t]{2}{*}{$\begin{array}{l}\text { Cup-to-disc ratio } \\
\text { difference of at least }\end{array}$} & \multirow[t]{2}{*}{$\begin{array}{l}\text { Side with the } \\
\text { larger optic cup }\end{array}$} & \multicolumn{2}{|c|}{$\begin{array}{l}\text { Side preferred } \\
\text { during sleep }\end{array}$} & \multirow[t]{2}{*}{$\begin{array}{l}\text { Probability of } \\
\text { agreement }\end{array}$} & \multirow[t]{2}{*}{$\begin{array}{l}\text { Agreement } \\
\text { (\%) }\end{array}$} \\
\hline & & & Right & Left & & \\
\hline \multirow[t]{2}{*}{ 1) } & $\geq 0.10$ & Right & 71 & 15 & $P=0.00 \mathrm{I}$ & 78 \\
\hline & & Left & 15 & 36 & $n=137$ & \\
\hline \multirow[t]{2}{*}{ 2) } & $\geq 0.15$ & Right & 47 & 8 & $P=0.001$ & 81 \\
\hline & & Left & 7 & 18 & $\mathrm{n}=80$ & \\
\hline \multirow[t]{2}{*}{ 3) } & $\geq 0.20$ & Right & 31 & 3 & $P=0.01$ & 85 \\
\hline & & Left & 5 & 13 & $n=52$ & \\
\hline \multirow[t]{2}{*}{ 4) } & $\geq 0.25$ & Right & 21 & 2 & $P=0.005$ & 84 \\
\hline & & Left & 3 & 5 & $n=31$ & \\
\hline
\end{tabular}

Notes: Data were subgrouped into those patients exhibiting a cup-to-disc ratio disparity $\geq 0.10,0.15,0.20$, and 0.25 . Agreement between the reported preferred side of eye dependency during sleep and the optic nerve with the larger cup-to-disc ratio was calculated for each subgroup. For patients observed to have at least a 0.10 cup-to-disc ratio asymmetry, there was a $78 \%$ agreement $(7 I+36 /$ total $)$. 


\section{Relationship of sleep preference to cup size difference}

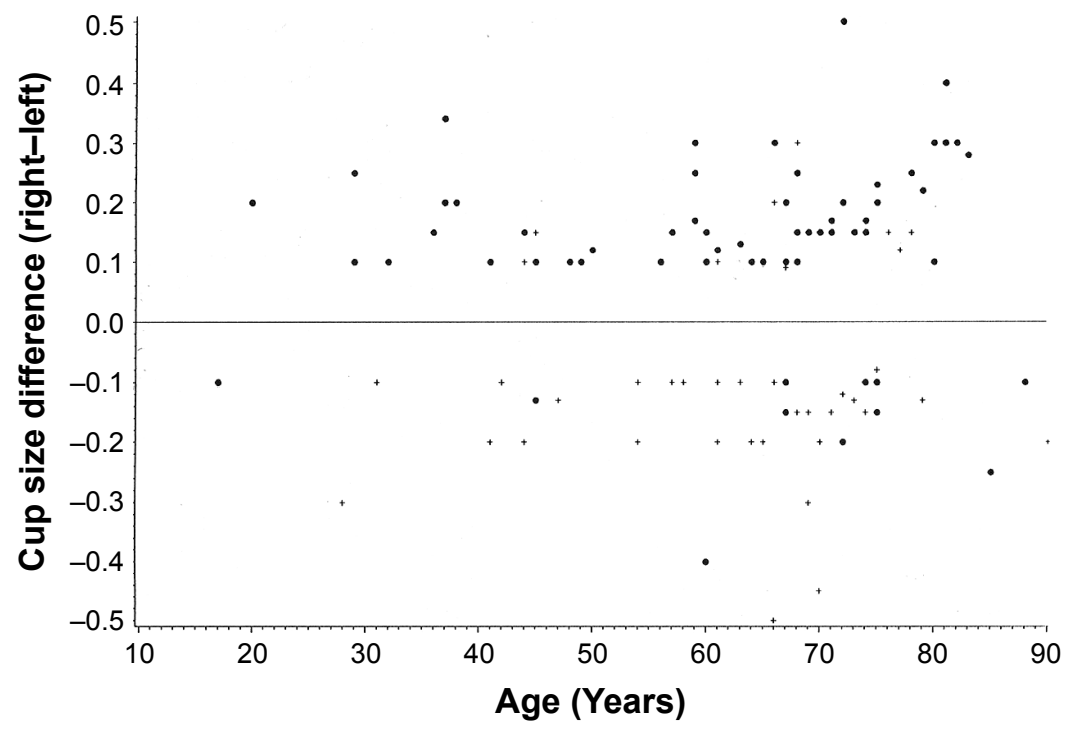

Figure 2 Graph of the vertical cup-to-disc ratio of the right eye minus the left eye, versus age, for patients observed to have at least a 0.10 cup-to-disc ratio asymmetry Notes: The eye with the larger optic cup coincided with the side of sleep preference $78 \%$ of the time, $P \leq 0.00$ I, $n=137$. Patients who reported they slept on their right side appear as $\bullet$ and those who preferred their left side as +. Patients with larger cup-to-disc ratios in the right eye who preferred a right-sided sleep posture therefore appear as a $\bullet$ above the horizontal and those with a larger cup-to-disc ratio in the left eye who preferred a left-sided sleeping posture appear as a + below the horizontal.

\section{Protocol 3, Experiment I}

In the third protocol of this paper, five surgeons attempted to produce a constant induced pressure that they felt was typical for them during their use of DOM. A typical tracing can be seen in Figure 3. Each of these compressions had a mean pressure value and range. Additionally, since four compressions were made by each surgeon, each surgeon had his own mean pressure and mean range of pressures for all four compressions, as well as variation around both of these means. All numerical data collected during DOM on human subjects are shown in Table 4. The mean IOP induced by all surgeons during this phase of the experiment was $104 \pm 8 \mathrm{mmHg}$ (SEM). The mean of the range of pressure for these pre-coached compressions was $17 \pm 3 \mathrm{mmHg}$ (SEM); the target range was $0 \mathrm{mmHg}$.

Subsequently, each surgeon received verbal cues during three more ocular compressions in order for the surgeon to acquire a tactile sensation for an induced pressure of $90 \mathrm{mmHg}$. After 5 minutes, the surgeons were asked to reproduce the $90 \mathrm{mmHg}$ pressure while no longer receiving any cues except their learned tactile sense. Each surgeon had three attempts to produce the $90 \mathrm{mmHg}$ target pressure. Reported results are the mean and SEM for the three attempts. The mean induced IOP for all surgeons after "training" to produce $90 \mathrm{mmHg}$ was $95 \pm 3 \mathrm{mmHg}$ (SEM; Table 4). The mean range of pressures after "training" for all surgeons decreased to $11 \pm 1 \mathrm{mmHg}$ (SEM).

\section{Discussion}

For the purposes of discussion, the term "ocular-pillow coupling" (OPC) will refer to the mechanical coupling of the closed eye with any surface during sleep and the potential increase in IOP attendant with it.

The data collected in this study support the hypothesis that IOP can be elevated to a clinically significant level during eye contact with a pillow during a side sleeping posture. The presence of respiratory variation, the progressive decrease in IOP measured with the OPE during the "sleep" session, and the unilateral decrease in IOP of the dependent compressed eye after the "sleep" session all substantiate the hypothesis that the weight of the head is transmitting pressure to the globe when physical contact is made with a pillow. The effects of neck muscle contraction cannot be negated and could either falsely depress or elevate the IOP; the neck muscle tone varies from flaccid in rapid eye movement sleep to active in lighter stages of sleep. ${ }^{23}$

The estimates of IOP during OPC may be affected by many variables. The device itself was calibrated manometrically just before each "sleep session". The plastic of the fluid-filled bladder was flexible but relatively inelastic. These properties were chosen to match those of the globe. When the two systems were physically adjacent and pressure was applied to one, the pressure of the fluid within both chambers became approximately equal. The ability of this device to estimate IOP was validated using a cannulated 


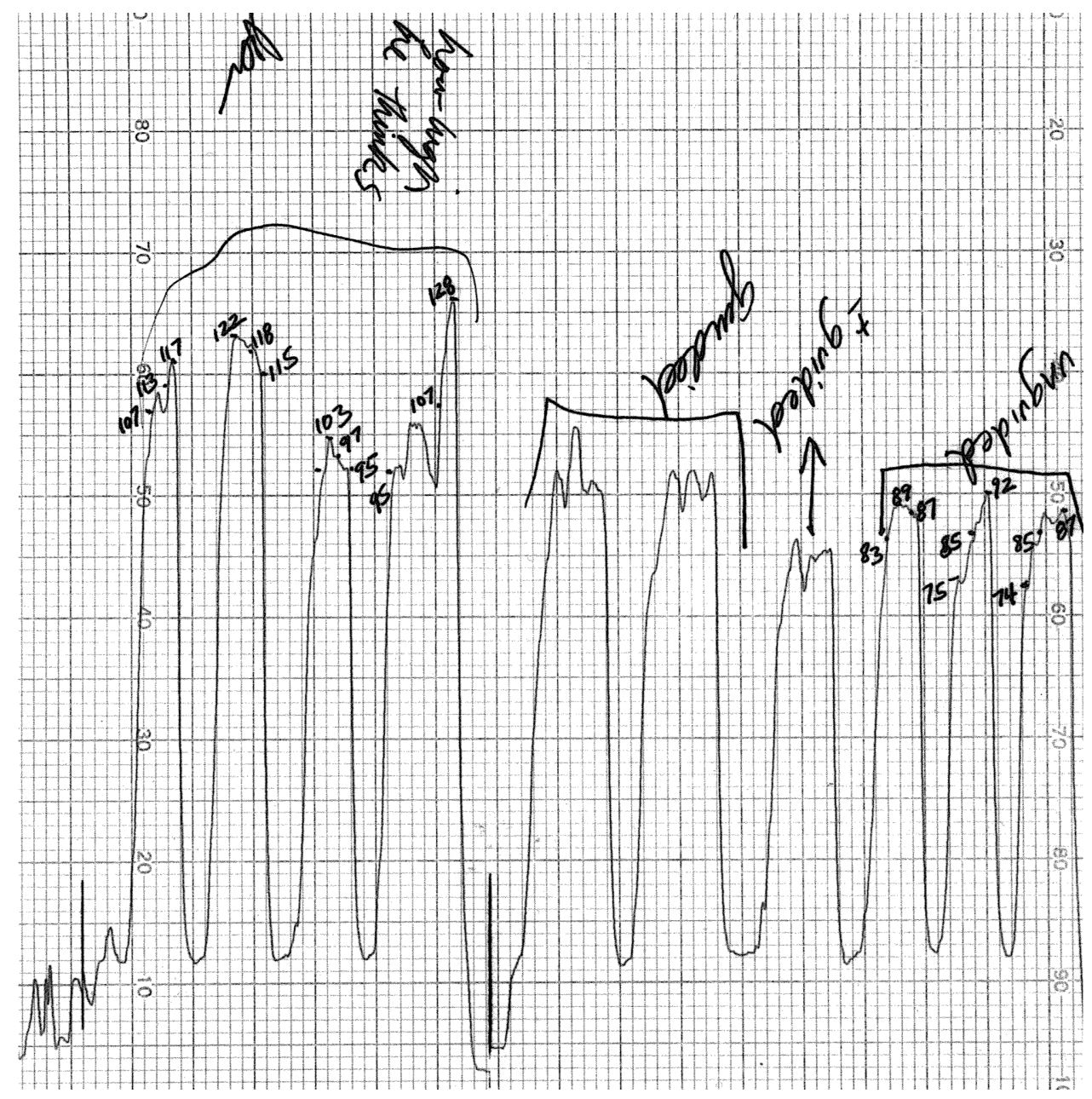

Figure 3 An original data tracing is shown that was produced while one glaucoma surgeon performed DOM in conjunction with the OPE.

Notes: The fluid-filled bladder was interposed between the surgeon's fingertips and the subject's closed eyelid. The numbers written on the graph are pressures in mmHg. The surgeon was first instructed to reproduce the magnitude of the compression they felt was typical for their ideal DOM maneuver while blinded to the IOPs they were inducing; from the left side of the tracing, this is the first four bracketed peaks labeled in cursive "how high he thinks". Each compression has a pressure range and as such, a time-weighted mean pressure value. Subsequently, each surgeon was "coached' while attempting to produce a target IOP of 90 mmHg (seen as the next two bracketed compressions labeled "guided" and a subsequent one labeled " \pm guided"). Another surgeon provided verbal feedback on the IOPs that were being produced while watching them on OPE's strip chart recorder. After 5 minutes, the newly "trained" surgeon was asked to reproduce this target pressure, without the benefit of coaching, for three more compressions (the last three peaks seen at the far right of the figure, bracketed, and labeled "unguided"). All DOM was performed on human subjects with normal IOPs.

Abbreviations: DOM, digital ocular massage; IOP, intraocular pressure; OPE, ocular pressure estimator.

human donor eye in a mock orbit and a cannulated pig eye, in vivo. ${ }^{1}$ It is, however, important to realize that the OPE, like other external methods for estimating IOP, is registering IOP in a perturbed system. Even though the plastic bladder was designed to be as thin as possible, it was still approximately $1.5 \mathrm{~mm}$ thick when it was used. This extra thickness meant that an individual's eye effectively came into contact with external surfaces sooner than it would have without the plastic fluid-filled bladder being interposed. It was likely that the perturbations induced by the OPE slightly raised the IOP, as do other applanating devices used to estimate IOP. The fact that the OPE only produced accurate estimates of the IOP does not, however, invalidate the conclusion that sleeping on one's eye can substantially elevate that eye's IOP.
The concept that a particular sleep posture could be pathologic to the eyes has precedent. The floppy eyelid syndrome ${ }^{24}$ is a condition that is typically manifest in obese patients. Patients with the floppy eyelid syndrome present with chronic ocular surface pathology that is the result of ocular contact with various surfaces incurred while sleeping. The eyelids in these patients are not anatomically and functionally able to maintain proper tension on the globe to prevent this chronic nocturnal trauma. It has been observed by authors describing this clinical entity that asymmetric findings in this condition are the result of sleep posture preferences that place the more injured eye in a dependent position..$^{24}$ In the initial description of this condition, six out of six patients who reported a sleep posture preference of one side over another had only ipsilateral ocular surface 
Table 4 Intraocular pressures induced during digital ocular massage

\begin{tabular}{|c|c|c|c|c|c|c|c|}
\hline \multirow[t]{2}{*}{ Surgeon } & \multicolumn{3}{|c|}{$\begin{array}{l}\text { Surgeons' ideal DOM induced pressure } \\
\text { (precoaching) }\end{array}$} & \multicolumn{4}{|c|}{$\begin{array}{l}\text { Reproducibility of targeted } 90 \mathrm{mmHg} \\
\text { (postcoaching) }\end{array}$} \\
\hline & Compression & Range & Mean & Compression & Range & Mean & Mean-90 \\
\hline \multirow[t]{6}{*}{ A } & I) & 10 & 95 & I) & 13 & 98 & 08 \\
\hline & 2) & 13 & 88 & 2) & 18 & 100 & 10 \\
\hline & 3) & 08 & 70 & 3) & 08 & 110 & 20 \\
\hline & 4) & 16 & 90 & & & & \\
\hline & Mean & 12 & 86 & & 13 & 103 & 13 \\
\hline & SEM & 02 & 06 & & 03 & 04 & 04 \\
\hline \multirow[t]{6}{*}{ B } & l) & 46 & 102 & I) & 34 & 107 & 17 \\
\hline & 2) & 21 & 93 & 2) & 08 & 69 & 21 \\
\hline & 3) & 48 & 122 & 3) & 03 & 117 & 27 \\
\hline & 4) & 21 & 126 & & & & \\
\hline & Mean & 29 & 111 & & 15 & 98 & 22 \\
\hline & SEM & 11 & 08 & & 10 & 15 & 03 \\
\hline \multirow[t]{6}{*}{ C } & I) & 08 & 93 & I) & 06 & 87 & 03 \\
\hline & 2) & 15 & 83 & 2) & 10 & 100 & 10 \\
\hline & 3) & 06 & 92 & 3) & 06 & 91 & 01 \\
\hline & 4) & 21 & 70 & & & & \\
\hline & Mean & 13 & 85 & & 07 & 93 & 05 \\
\hline & SEM & 03 & 05 & & 01 & 05 & 03 \\
\hline \multirow[t]{6}{*}{$D$} & I) & 10 & 113 & I) & 06 & 87 & 03 \\
\hline & 2) & 07 & 118 & 2) & 17 & 85 & 05 \\
\hline & 3) & 08 & 97 & 3) & 13 & 85 & 05 \\
\hline & 4) & 32 & 107 & & & & \\
\hline & Mean & 14 & 109 & & 12 & 86 & 04 \\
\hline & SEM & 06 & 05 & & 03 & $0 \mathrm{I}$ & 01 \\
\hline \multirow[t]{6}{*}{$\mathrm{E}$} & I) & 19 & 136 & I) & 06 & 105 & 15 \\
\hline & 2) & 26 & 130 & 2) & 08 & 91 & 01 \\
\hline & 3) & 16 & 127 & 3) & 17 & 85 & 05 \\
\hline & 4) & 16 & 113 & & & & \\
\hline & Mean & 19 & 127 & & 10 & 94 & 07 \\
\hline & SEM & 02 & 05 & & 03 & 06 & 04 \\
\hline Mean of mean & & $17 \pm 3$ & $104 \pm 8$ & & $I I \pm I$ & $95 \pm 3$ & $10 \pm 3$ \\
\hline Mean of SEMs & & $5 \pm 2$ & $6 \pm 1$ & & $4 \pm 2$ & $6 \pm 2$ & $3 \pm 1$ \\
\hline
\end{tabular}

Notes: All pressures are in $\mathrm{mmHg}$. Each individual surgeon had a range and time-weighted mean IOP for their pre- and postcoaching compressions. The absolute value of the difference between the $90 \mathrm{mmHg}$ target pressure and the pressure actually produced during postcoaching massage is shown in the last column, and shows how close the surgeon came to reproducing the target pressure. Statistics on the entire group of surgeons are shown at the bottom of the table.

Abbreviations: IOP, intraocular pressure; DOM, digital ocular massage; SEM, standard error of mean.

pathology; only historical patient reports were utilized. All patients who manifested unilateral ocular surface pathology had relief of their symptoms within 3 weeks after using a rigid shield over their eye. The significant improvement of signs and symptoms after this intervention strongly suggested that the mechanism of damage in this condition was the result of OPC.

The tracings obtained during the simulated "sleep" sessions are similar to tonographic results. The dynamics observed in the "sleep" tracings, however, result from both the pressure-sensitive outflow of aqueous humor from the anterior chamber and the decompression of the orbit proper from the extra weight of the head that is transferred to the orbit during OPC. It is therefore likely that patients with poor trabecular meshwork outflow facility would take longer and be less effective in decompressing their globes when the constant weight of the head was applied to the orbit. However, since orbital decompression was measured simultaneously, this tracing was not as selective a measure of outflow facility as tonography. In most cases, the graph of IOP during OPC rose initially, slowly decreased during the "sleep session", and tended to approach a new plateau level. The clinically relevant features of this graph are the time it takes to reach this new relatively stable IOP and the IOP magnitude of the plateau itself; both components contribute to the area under the IOP-time curve. 
Orbital decompression in this model represents the decompression of the globe plus the cushioning effect of the orbital fat and ease of blood displacement from the bony orbit itself. It is likely that patients with an orbital infiltrative process such as thyroid ophthalmopathy would have a slower and less effective orbital decompression ${ }^{25}$ with OPC.

In general, the bony orbital rim should protect the globe from externally applied pressure unless the object that makes contact with the eye is protuberant, (eg, a hand) or is moldable (eg, a pillow). Similarly, it was expected that patients with higher readings on exophthalmometry would be at greater risk from this effect. However, in our ocular normotensive subjects with exophthalmometry readings in the normal range, there was no apparent relationship between the sleep tracings and ocular protuberance. Perhaps abnormally protuberant eyes would show a more definite correlation. The higher incidence of glaucoma in the African-American population may be in part related to the phenomenon of OPC; African-Americans have a racial ocular prominence that may be up to $2 \mathrm{~mm}$ greater than Caucasians before it is considered abnormal. ${ }^{26}$

When considering the potential mechanisms of damage from OPC, several possibilities can be entertained. The typical fluctuations of aqueous humor flow and IOP occur slowly, and the eye has an opportunity to autoregulate its functions. With the pressure elevations that occurred during OPC, the change in IOP was much faster, leaving less chance for autoregulation to be effective. In addition, frequent and substantial changes in IOP may be more deleterious than gradual pressure changes of the same magnitude. Even though the periods of significant IOP elevation during OPC may last only a few minutes, when considered chronically, such relatively short periods may represent a major portion of the time during which an eye is exposed to damaging pressures.

To properly evaluate the perfusion of the optic nerve head and globe, considerations must be paid not only to the IOP but also to the systemic blood pressure as well. There is evidence that there are significant decreases in systemic blood pressure during sleep in normal subjects. ${ }^{27}$ Drops in nocturnal blood pressure in glaucoma patients exceed those reported for healthy young adults. ${ }^{28,29}$ Patients with progressive visual field deterioration have statistically significantly lower blood pressure indices when compared to patients without visual field deterioration. ${ }^{28,29}$ Nocturnal hypotension is a likely contributor to the multifactorial insults that result in optic nerve morbidity.

Many ocular diseases are likely to be affected by the combined effects of OPC and nocturnal hypotension. Patients with glaucoma, who typically have poor outflow facility, a weakened optic nerve structure, and abnormal optic nerve head vascular autoregulation, ${ }^{30}$ may be especially likely to incur damage from these phenomena. These occult IOP elevations may also explain some cases of "low-tension" or "normal tension glaucoma", 31,32 of clinically worsening glaucoma in the face of repeatedly low office-measured IOPs, and of asymmetric glaucoma with reproducibly equal IOPs in the office. Studies of the natural history of low-tension glaucoma have not fully explained asymmetries in optic nerve cupping and visual field defects based on corresponding asymmetries in the office-measured IOP. ${ }^{31,32}$ The phenomenon of OPC may help to explain the pathogenesis of these asymmetric findings.

In one "normal" volunteer, the IOP measuring during "sleep" averaged $57 \mathrm{mmHg}$ and peaked at $100 \mathrm{mmHg}$. This subject was quite comfortable during the time that his IOP was $100 \mathrm{mmHg}$. Ocular anesthesia from topical eyedrops was an unlikely explanation for this comfort since the "sleep session" was started 1 hour after the last topical medication. IOPs in the range of $80-100 \mathrm{mmHg}$ were similarly found to be comfortable in subjects experiencing DOM performed in conjunction with the OPE. These levels of IOP are greater than many people's diastolic blood pressure. Furthermore, they have been shown to impair axoplasmic flow in the optic nerve, ${ }^{33,34}$ mechanically deform the optic nerve head, ${ }^{35,36}$ impair prelaminar ${ }^{37}$ and postlaminar ${ }^{38}$ optic nerve perfusion, and decrease the amplitude of the visual evoked potential. ${ }^{30}$ This young individual, with normal baseline eye and blood pressure, was subsequently observed to have glaucomatous cupping in both eyes and an early visual field defect that corresponded to a notch in the optic cup. This patient consistently slept on his sides and did not have a side preference. In routine practice, this patient would be diagnosed as having low-tension glaucoma.

The likely effect of simultaneous nocturnal hypotension and elevations of IOP from external compression is a decrease in ocular perfusion and/or and increased likelihood of rheologic turbulence. As such, OPC may have an adverse effect on any ocular disease that depends upon adequate perfusion. During the course of this study, we interviewed three patients who went to bed with normal vision and awoke with a retinal venous occlusion; all three patients stated that they preferred to sleep on the side of their vascular occlusion when asked the standard set of questions. In the recently described orbital infarction syndrome, ${ }^{39}$ two of the three cases described initiation of, or a substantial decrement of visual function upon awakening; OPC may have played a role in these cases. OPC may be related to the pathogenesis of acquired optic nerve pits, ${ }^{40,41}$ which are acquired over a short period of time 
and are unilateral or bilateral, but acquired sequentially. Diabetic and hypertensive retinopathy are likely candidates for increased morbidity from OPC and nocturnal hypotension. These occult and chronic IOP transients may even influence the structural integrity of Bruch's membrane and the natural history of age-related macular degeneration. Finally, OPC and nocturnal hypotension may affect a large subset of the population at all ages and may result in an accelerated axonal loss from the optic nerve, even in apparently normal eyes; the calculated "normal" rate of optic nerve axon loss of approximately $4,000^{42}$ to 5,600 axons $^{43}$ per year may be adversely influenced by these phenomena.

A separate pathologic response to OPC may be the induction of the oculo-cardiac reflex. This reflex can be induced by ocular compression ${ }^{44}$ and its activation produces a vagally mediated brady-dysrhythmia that can cause fatal cardiac arrest. ${ }^{45}$ There is increased vulnerability of the heart to dysrhythmias during sleep ${ }^{46}$ and unconscious patients show a greater oculo-cardiac reflex than awake patients. ${ }^{44}$ The prone position of sleep, a position of risk for OPC, is associated with an increased risk of sudden infant death syndrome. ${ }^{47}$ The evidence implicating OPC as a potential contributor to the pathogenesis of some cases of sudden infant death syndrome is compelling and merits further study.

In considering the possible treatment of OPC, it would be expected that any device that transmits the forces of the head to the bony orbit could protect the globe from damage. The rigid Fox shield has been shown to be effective for postoperative protection and for treating the floppy eyelid syndrome. ${ }^{24}$ A similar device protecting both eyes should provide necessary protection. A device that provides this binocular protection has been invented by one of the authors (MSK). Currently, he is recommending rigid shields as an adjunctive low morbidity treatment for functionally one-eyed glaucoma patients who have little reserve in their remaining eye and who report sleep postures other than strictly supine.

Our preliminary data suggest that the ocular compressions produced during normal sleeping postures can lead to substantial elevations of IOP. If the IOPs measured in this study were obtained in the office, they would likely be considered a risk factor for ocular pathology. The association between the side with the larger cup-to-disc ratio and the side with eye dependency during sleep may be causal. This study was not designed to identify causality or to address incidence. Further research, which is designed to address causality and to address incidence, and incorporates fundus photography, biometry, and sleep monitoring, is warranted, considering the broad implications of these findings; fortunately, this mechanism for ocular damage is most likely preventable.
The purpose of trying to identify and characterize patients with a sleep posture preference was to test whether chronic exposure to occult intermittent IOP elevations on one side might produce objective evidence of anatomic damage which was correspondingly asymmetric. To test this hypothesis, we specifically selected patients with asymmetry of both optic nerve cupping and of sleep posture preference. We would observe, however, that the concept of potential eye damage from OPC is not limited to damage on one side.

Possible sources of error in the cup-to-disc ratio asymmetry arm of the study include the estimates of the cup-to-disc ratios themselves and the sleep preferences reported by the patients. In this pilot study, one author (MSK) was the sole judge of the vertical cup-to-disc ratios. When the margin of the disc was sloping or ambiguous, the patient was not asked about sleep preference and was excluded from this study. It was felt that cup-to-disc ratio asymmetry of greater than or equal to 0.1 could be reliably identified using the standard methods described. Intra-observer and inter-observer variability are important to consider when the cup-to-disc ratio is estimated. ${ }^{48}$ For this reason, this pilot study relied upon only one final quantifier of the cup-to-disc ratios; interobserver variability was thus eliminated.

Because larger degrees of cup-to-disc ratio asymmetry are more obviously identified as asymmetric, the statistics were applied to the data in subgroups. In the group that contained patients with at least a 0.10 vertical cup-to-disc ratio asymmetry, there were 137 patients; $100 \%$ of the patients had at least a 0.10 vertical cup-to-disc ratio asymmetry (Table 1). There were 80 patients $(58 \%)$ in the group with at least a 0.15 difference, 52 patients $(40 \%)$ with at least a 0.20 difference, and 31 patients $(23 \%)$ with at least a 0.25 difference. This grouping was designed to de-emphasize the importance of absolute cup-to-disc ratio quantification. The persistence of statistical significance with larger degrees of cup-to-disc ratio asymmetry helps to alleviate concerns regarding intraobserver quantification of these cup-to-disc ratios. The persistence of statistical significance in all four of these subgroups further substantiates the association of the side with the larger optic cup and the reported side of sleep preference. In projected future studies, stereoscopic disc photographs and masked readers will be used to add additional reliability to the cup-to-disc ratio measurements.

Another potential source of error in assessing cup-to-disc ratios was the assumption that the observed asymmetries were acquired. Patients with substantial axial ammetropia are known to manifest asymmetries in scleral canal size. This could result in apparent cup-to-disc ratio asymmetry even though there may be the same number of axons in both optic 
nerve heads. Patients without axial ammetropia are less likely to manifest large differences in scleral canal size. In this pilot study, patients were excluded if they had greater than $2 \mathrm{D}$ of presurgical ammetropia. This was done to limit the possibility of cup-to-disc ratio asymmetry arising from the differences in scleral canal size. Biometric estimates of axial length and of scleral canal size could help identify this potential source of inherent cup-to-disc ratio asymmetry.

The concern of experimental bias must be raised. The judge of the cup-to-disc ratios and the sleep history interviewer was the same investigator. However, since the cup-to-disc ratios were first quantified and their values committed on paper, the only bias that could be applied to the experimental outcome would be one in which the experimenter encouraged the patients' reported sleep preference to coincide with the observed cup-to-disc ratio asymmetry. For this reason, a standard set of questions (described earlier) was asked each time and the qualifier, "you do not have to have a favorite side", was included. The patients had no idea what the physician's expectations were or how they should answer to please their physician. This is a fundamentally different situation from inquiries into compliance with topical drug use. ${ }^{49}$ In the compliance studies, the patients clearly had the bias to answer that they had used their drops as directed. In summary, for this study, the experimenter had no bias in quantifying the cup-to-disc ratios before assessing sleep posture preference, and the patients had no bias in answering the examiner's questions regarding sleep posture preferences.

It is important to note that people exhibit a wide spectrum of sleep behaviors and postures that are maintained for different lengths of time and vary for a given individual from night to night. Sleep laboratory research has shown that healthy people in their second and third decades of life maintain a given sleep posture for an average of 15 minutes, ${ }^{50}$ while healthy patients in the seventh and eighth decades of life maintain their sleep positions for an average of 33 minutes. ${ }^{20}$ In another sleep laboratory study, ${ }^{51}$ it was shown that among healthy sleepers of many ages, about half of all postures were held for less than 5 minutes, about a fifth were held for 5-10 minutes, about a tenth were held for 10-15 minutes, and less than once a night a given posture remained unchanged for as long as an hour. The existence of illness, usually cardiorespiratory or orthopedic, ${ }^{20,21}$ can influence the type and duration of sleep postures. The presence of a bed partner also influences the range of sleep positions. ${ }^{51}$

A few researchers have determined the concordance between patients' report of their generally preferred sleep posture with objective observations of other tests of reliability. One author ${ }^{19}$ found that individuals can very accurately determine their generally preferred sleep position and used bed partners as an objective means of corroboration; it was this information that led us to exclude patients who reported different sleeping postures from their spouses. In another study, ${ }^{52} 51$ healthy young women were given photographs of sleeping positions to choose from in identifying their own preference; many of these were quite similar. Six months later, the same women were asked to make the same choice, and 46 of the 51 women chose the identical or highly similar position twice. Another study ${ }^{53}$ assessed reported sleep postures and compared them to direct intermittent observations at night. Among the 207 nonpregnant controls, there was excellent correlation between the reported postures and the postures observed during the initial hours of sleep. Later in the sleep cycle, there was a substantial abandonment of the supine position in favor of the Sims' position (a side position that places the eye at risk for OPC) and the prone position. The percentage of patients observed in the lateral position was consistent throughout the night $(41.2 \%)$ and very similar to the $43.9 \%$ who claimed to sleep in this position. Although the data did not address the correlation between the reported and observed side of sleep, they seem to indicate that a reported preference for side sleeping in general is relatively reliable; supine postures, although initially assumed, tended to be less consistently maintained through the night. In the initial report of the floppy eyelid syndrome, ${ }^{24}$ the authors used patient-reported sleep postures in their correlations of sleep behavior and clinical pathology. Other researchers have found the correlation between reported sleep posture and sleep laboratory observations to be less reliable. ${ }^{51}$ A minority of people volunteered that they had a side preference when simply asked if they slept on their backs, tummy, or side. These patients usually had a coexisting medical condition ${ }^{20,21}$ that necessitated this posture, and these patients were felt to give the most reliable data concerning sleep posture. Most people related their impressions of how they slept and this is inherently not as accurate. Even though there are several potential sources of error in assessing sleep posture preferences through patient self-reporting, an unbiased questioning strategy, an insistence on spousal corroboration, and a sufficiently large sample size limit this potential in this study.

Among our patients who reported a side preference for sleeping, $63 \%$ of them reported a right-sided sleep preference. This preference for sleeping on the right side in preference to the left has been previously observed ${ }^{21,50}$ in sleep laboratory studies. This strengthens the reliability of the sleep posture data in this study. The preference for sleeping on the right 
side may be related to the finding of zones of greater cup depth and pallor in the right eyes of ocular hypertensives with symmetrical office-measured IOPs. ${ }^{54}$

An underlying assumption of this study is that observed cup-to-disc ratio asymmetries represent acquired differences. ${ }^{55}$ It would, therefore, be expected that if a given sleep pattern was consistent throughout a person's life, the cup-to-disc ratio disparity should increase with increasing age. A statistically insignificant trend was observed in the magnitude of cup-to-disc ratio disparity with increasing age, but it was accompanied by an increase in this parameter's variability as well. The lack of statistical significance was felt to be largely due to an inadequate sample size of people aged less than 40 years.

The paucity of young subjects exhibiting cup-to-disc ratio asymmetry of at least 0.1 was in part secondary to fewer contacts with this age group. However, most patients who possess an acquired cup-to-disc ratio disparity probably evolved to this state over time from cup-to-disc ratio symmetry. Since this evolution may take decades to produce a cup-to-disc ratio asymmetry of at least 0.1 , a young person would be more likely to be in a subclinical transition from cup-to-disc ratio symmetry. This may be another reason why there were fewer young patients identified who met this study criterion. It was our impression that there was a lower incidence of unambiguous cup-to-disc ratio asymmetry in the younger population although no incidence data were collected in this pilot study. Future studies will address the incidence of cup-to-disc ratio asymmetry in different age groups and the incidence of cup-to-disc ratio asymmetry among people who sleep on their backs.

DOM is one of several methods available to promote aqueous drainage in the postoperative period after glaucoma filtering surgery. When successful, the filtration bleb expands and the IOP falls; adherents of the technique believe that this short-term result, particularly when repeated at regular intervals, can lead to long-term improvement in drainage function. Until now, there has been no means to quantify the pressure applied, so questions of efficacy may partly represent variability in technique.

There are several benefits that may be derived from quantifying this procedure. There is likely to be a range of digital pressures that, in responsive eyes, will produce bleb expansion and IOP lowering. It would be useful to define this range. If the range is narrow, the OPE could be used to train doctors and patients (as we did in this pilot study) to produce this effective pressure. If the range is broad (as we suspect), it may be necessary to determine the effective pressure for each individual patient by in-office testing, and then either train the patient to produce that pressure in the office, or send the patient home with an OPE to allow direct monitoring of each application. We have configured one prototype of the OPE to be portable for patient use at home. After the surgeon has established the range of induced pressures that are effective for a given patient, this form of the OPE can be set so that an auditory and visual signal indicates when the patient has achieved the targeted pressure. Customizing the pressure applied to the requirements of each patient should enhance the safety of the procedure. The OPE could also be configured to digitally record the time the patient performed the DOM, how long each compression lasted, and the magnitude of the induced pressures. Recording each treatment may improve patient compliance for doing DOM.

\section{Acknowledgment}

This work is supported in part by an unrestricted grant from Research to Prevent Blindness.

This paper and its tandem publication ${ }^{1}$ represent the culmination of the work that was presented to the glaucoma community at ARVO, ${ }^{56,57}$ where the concepts, methods, and clinical implications for estimating the IOP during external ocular compression were first shared.

\section{Disclosure}

The authors report no conflicts of interest in this work.

\section{References}

1. Korenfeld MS, Dueker DK. Noninvasive estimation of the intraocular pressure produced during ocular compression. Clin Ophthalmal. 2015;9:71-77.

2. Ericson LA. Twenty-four hourly variations on the aqueous flow: examination with peripheral suction cup. Acta Ophthalmol Suppl. 1958; 37(Suppl 50):1-95.

3. Reiss GR, Lee DA, Topper JE, Brubaker RF. Aqueous humor flow during sleep. Invest Ophthalmol Vis Sci. 1984;25(6):776-778.

4. Topper JE, Brubaker RF. Effects of timolol, epinephrine, and acetazolamide on aqueous flow during sleep. Invest Ophthalmol Vis Sci. 1985;26(10):1315-1319.

5. Tadayoshi I, Godi T, Yoshiaki K. Diurnal variation of intraocular pressure of normal-tension glaucoma. Influence of sleep and arousal. Ophthalmology. 1991;98(3):296-300.

6. Framptom P, Rin DD, Brown B. Diurnal variation of intraocular pressure and the overriding effects of sleep. Am J Optom Physiol Optics. 1987;64(1):54-61.

7. Oshika T, Araie M. The mechanism of diurnal variation of aqueous protein concentration. Invest Ophthalmol Vis Sci [Suppl]. 1991; 32:887.

8. Hollenhorst RW, Svien HJ, Benoit CF. Unilateral blindness occurring during anesthesia for neurosurgical operations. Arch Ophthalmol. 1954;52(6):819-830.

9. Dodden ED, Gibralter RP, Udell IJ, Perry HD. Keratoconus associated with floppy eyelid syndrome. Ophthalmology [Suppl]. 1990; 97:139.

10. Epstein DL. Chandler and Grant's Glaucoma. 3rd ed. Philadelphia, PA: Lea \& Febiger; 1986:430. 
11. Fitzgerald JR, McCarthy JL. Surgery of the filtering bleb. Arch Ophthalmol. 1962;68:453-467.

12. Heilmann K, Richardson KT. Glaucoma, Conceptions of a Disease, Pathogenesis, Diagnosis, Therapy. Stuttgart, FRG: Georg Thieme Verlag; 1978:360.

13. Kolker AE, Hetherington J Jr. Becker-Shaffer's Diagnosis and Therapy of the Glaucomas. 5th ed. St Louis, MO: C.V. Mosby Company; 1983:465.

14. Luntz MH, Harrison R, Schenker HI. Glaucoma Surgery. Baltimore, MD: Williams \& Wilkins; 1984:81, 85.

15. MacRae SM, Van Buskirk EM. Late wound dehiscence after penetrating keratoplasty in association with digital massage. Am J Ophthalmol. 1986;102:391-392.

16. Parrow KA, Shin DH. Enhancing filtration in the early postoperative trabeculectomy refractory to digital massage. Ophthalmic Surg. 1990; 21(6):401-403.

17. Segrest DR, Ellis PP. Iris incarceration associated with digital ocular massage. Ophthalmic Surg. 1981;12(5):349-351.

18. Sugar HS. Successful filtering blebs. Ann Ophthalmol. 1971;3(5): 485-487.

19. Dunkell S. Sleep Positions: The Night Language of the Body. 1 st ed. New York, NY: William Morrow \& Co., 1997:10.

20. Lorrain D, De Koninck J, Dionne H, Goupil G. Sleep positions and postural shifts in elderly persons. Percept Mot Skills. 1986;63(2 Pt 1): 352-354.

21. Cartwright RD. Effect of sleep position on sleep apnea severity. Sleep. 1884;7(2):110-114.

22. Siegel S. Non-Parametric Statistics. New York, NY: McGraw-Hill; 1956:61.

23. Kelly DD. Sleep with rapid eye movements. In: Kandel ER, Schwartz JH, editors. Principles of Neural Science. New York, NY: Elsevier North Holland, Inc.; 1981:475.

24. Culbertson WW, Ostler HB. The floppy eyelid syndrome. Am J Ophthalmol. 1981;92(4):568-575.

25. Frueh BR. Graves' eye disease: orbital compliance and other physical measurements. Trans Am Ophthalmol Soc. 1984;82:492-598.

26. Migliori ME, Gladstone GJ. Determination of the normal range of exophthalmometric values for black and white adults. Am JOphthalmol. 1984;98(4):438-442.

27. Somers VK, Dyken ME, Mark AL, Abboud MF. Sympathetic-nerve activity during sleep in normal subjects. $N$ Engl J Med. 1993;328(5): 303-307.

28. Hayreh SS, Zimmerman MB, Podhajsky P, Alward WL. The role of nocturnal hypotension in ocular and optic nerve ischemic disorders. Invest Ophthalmol Vis Sci [Suppl]. 1993;34:994.

29. Graham SL, Drance SM, Wijsman K, Mikelberg FS, Douglas GR. Nocturnal hypotension in glaucoma patients. Invest Ophthalmol Vis Sci [Suppl]. 1993;34:1286.

30. Pillunat LE, Stodmeister R, Wilmann I, Christ T. Autoregulation of ocular blood flow during changes in intraocular pressure. Preliminary results. Graefes Arch Clin Exp Ophthalmol. 1985;223(4):219-223.

31. Crichton A, Drance SM, Douglas GR, Schulzer M. Unequal intraocular pressure and its relation to asymmetric visual field defects in low tension glaucoma. Ophthalmology. 1989;96:1312.

32. Haefliger IO, Hitchings RA. Relationship between asymmetry of visual field defects and intraocular pressure difference in untreated normal (low) tension glaucoma population. Acta Ophthalmol (Copenh). 1990;68(5):564-567.

33. Quigley HA, Flower RW, Addicks EM, McLeod DS. The mechanism of optic nerve damage in experimental acute intraocular pressure elevation. Invest Ophthalmol Vis Sci. 1980;19(5):505-517.

34. Minckler DS, Tso MO, Zimmerman LE. A light microscopic, autoradiographic study of axoplasmic transport in the optic nerve head during ocular hypotony, increased intraocular pressure, and papilledema. Am J Ophthalmol. 1976;82(5):741-757.
35. Coleman AL, Quigley HA, Vitale S, Dunkelberger G. Displacement of the optic nerve head by acute changes in intraocular pressure in monkey eyes. Ophthalmology. 1991;98(1):35-40.

36. Levy ND, Crapps EE. Displacement of optic nerve head in response to short-term intraocular pressure elevation in human eyes. Arch Ophthalmol. 1984;102(5):782-786.

37. Ernest JT. Optic Disc blood flow. Trans Ophthalmol Soc U K. 1976; 96(3):348-351

38. Nitta A, Kimura Y, Takayma H. The blood flow in post-laminar optic nerve in monkeys was dependent on intraocular pressure level. Nippon Ganka Gakkai Zasshi. 1989;93(2):206-214.

39. Borruat FX, Bogousslavsky J, Uffer S, Klainguti G, Schatz NJ. Orbital infarction syndrome. Ophthalmology. 1993;100(4):562-568.

40. Javitt JC, Spaeth GL, Katz J, Poryzees E, Addiego R. Acquired pits of the optic nerve. Ophthalmology. 1990;97(8):1038-1043.

41. Lichter PR, Henderson JW. Optic nerve infarction. Trans Am Ophthalmol Soc. 1977;75:103-121.

42. Schmidt AM, Jonas JB, Schlotzer-Schrehardt UM, Naumann GOH. Human optic nerve fiber count and optic disc size. Invest Ophthalmol Vis Sci [Suppl]. 1992;33(6):2012-2018.

43. Balazsi AG, Rootman J, Drance SM, Schulzer M, Douglas GR. The effect of age on the nerve fiber population of the human optic nerve. Am J Ophthalmol. 1984;97(6):760-766.

44. Aschner B. Ueber einen bisher noch nicht beschriebenen Reflex vom Auge auf Kreislauf und Atmung. Verschwinden des Radialispulses bei Druck auf das Auge. Wien Klin Wochenschr. 1908;21:1529.

45. Sorenson BJ, Gilmore JE. Cardiac arrest during strabismus surgery. Am J Ophthalmol. 1956;41:748.

46. Froggatt P, James TN. Sudden unexpected death in infants. Evidence of a lethal cardiac arrhythmia. Ulster Med J. 1973;42(2):136-152.

47. Stanley FJ, Byard RW. The association between the prone sleeping position and sudden infant death syndrome (SIDS): an editorial review. J Paediatr Child Health. 1991;27(6):325-328.

48. Lichter PR. Variability of expert observers in evaluating the optic disc. Trans Am Ophthalmol Soc. 1976;74:532-572.

49. Kass MA, Melzer DW, Gordon M, Cooper D, Goldberg J. Compliance with topical pilocarpine treatment. Am J Ophthalmol. 1986;101(5):515-523.

50. De Koninck J, Gagnon P, Lallier S. Sleep positions in the young adult and their relationship with the subjective quality of sleep. Sleep. 1983;6(1):52-59.

51. Johnson HM, Swan TH, Weigand GE. In what positions do healthy people sleep? JAMA. 1930;94(26):2058-2062.

52. Domino G, Bohn SA. Hypnagogic exploration: sleep positions and personality. J Clin Psychol. 1980;36(3):760-762.

53. Ogita S, Imanaka M, Takebayashi T, et al. Significance of exercise and bed rest in pregnancy: study on the lying postures of gravidas during sleep. Ann Physiol Anthrop. 1990;9(2):93-98.

54. Raujo D, Schwartz B, Takamoto T. The relationship between the rate of change of visual field in the initial measurement of optic disc pallor and cupping. Invest Ophthalmol Vis Sci [Suppl]. 1988;29:355.

55. Caprioli J, Boeglin RJ, Hoffman D. Disc asymmetry in normal and glaucomatous populations. Invest Ophthalmol Vis Sci [Suppl]. 1991;32:718.

56. Korenfeld MS, Dueker DK. An apparatus for measuring digital pressure on the eye [ARVO Abstract 383]. Invest Ophthalmol Vis Sci. 1991;32(suppl):743.

57. Korenfeld MS, Dueker DK. Occult intraocular pressure elevations and optic cup asymmetry: sleep posture may be a risk factor. [ARVO Abstract 1433]. Invest Ophthalmol Vis Sci. 1993;34(suppl):994. 
Clinical Ophthalmology

\section{Publish your work in this journal}

Clinical Ophthalmology is an international, peer-reviewed journal covering all subspecialties within ophthalmology. Key topics include: Optometry; Visual science; Pharmacology and drug therapy in eye diseases; Basic Sciences; Primary and Secondary eye care; Patien Safety and Quality of Care Improvements. This journal is indexed on

PubMed Central and CAS, and is the official journal of The Society of Clinical Ophthalmology (SCO). The manuscript management system is completely online and includes a very quick and fair peer-review system, which is all easy to use. Visit http://www.dovepress.com/ testimonials.php to read real quotes from published authors. 